\title{
BOUNDS FOR THE SOLUTIONS OF A CLASS OF NONLINEAR PARTIAL DIFFERENTIAL EQUATIONS
}

ZEEV NEHARI ${ }^{1}$

1. Let $\Delta=\partial^{2} / \partial x_{1}^{2}+\cdots+\partial^{2} / \partial x_{n}^{2}$ denote the $n$-dimensional Laplace operator and let the symbols $D_{r}$ and $S_{r}$ stand for the open sphere $x_{1}^{2}+\cdots+x_{n}^{2}<r^{2}(r>0)$ and its boundary $x_{1}^{2}+\cdots+x_{n}^{2}=r^{2}$, respectively. We are concerned here with functions $u=u(P) \quad\left(P \in D_{R}\right)$ which are of class $C^{2}$ in $D_{R}$ and satisfy there the differential equation

$$
\Delta u=f(u) \text {, }
$$

or, more generally, the differential inequality

$$
\Delta u \geqq f(u) \text {. }
$$

In the literature on the subject $[1 ; 2 ; 3 ; 5 ; 7 ; 8]$, two closely related problems are investigated:

(a) What are the conditions to be imposed on the function $f(u)$ in order to guarantee the existence of a bound $\phi(r)=\phi(r, R ; f)$ such that

$$
\underset{P \in S_{r}}{u(P)} \leqq \phi(r, R ; f)
$$

if $u$ satisfies (2) in a region $D_{R}$ with $R>r$ ?

(b) Under what conditions on $f(u)$ will (2) have no solutions which are of class $C^{2}$ in the entire $n$-space?

Clearly, the nonexistence of such solutions is assured whenever it can be shown that $\phi(0, R ; f) \rightarrow-\infty$ for $R \rightarrow \infty$.

The most general conditions on $f(u)$ for which the existence of such bounds for the solutions of (2) have been established are $[3 ; 5]$ : $f(u)>0, f^{\prime}(u) \geqq 0$ for $-\infty<u<\infty$,

$$
\int_{0}^{\infty}\left[\int_{0}^{u} f(t) d t\right]^{-1 / 2} d u<\infty .
$$

In fact, if $f(u)>0$ and $f^{\prime}(u) \geqq 0$, condition (4) is both necessary and sufficient. It was also shown in [3] and [5] that the problem $u(P)$ $=\max$ is solved by a spherically symmetric solution $\phi(r)$ of (1) for which $\phi(r) \rightarrow \infty$ for $r \rightarrow R$, i.e., by a solution of the ordinary differential equation

Received by the editors June 21, 1962.

${ }^{1}$ This research was supported by Air Force Office of Scientific Research. 


$$
\phi^{\prime \prime}(r)+\frac{n-1}{r} \phi^{\prime}(r)=f(\phi)
$$

for which $\phi^{\prime}(0)=0$ and $\phi(r) \rightarrow \infty$ for $r \rightarrow R$. In those cases in which this solution can be found explicitly it is thus possible to determine the exact upper bound (3). An example is the two-dimensional equation $\Delta u=e^{u}$, which has the well-known solution

$$
u=2 \log \frac{\sqrt{ } 8 R}{R^{2}-r^{2}} .
$$

It was pointed out by Osserman [5] that an upper bound for $u(P)$ is given by any spherically symmetric function $v$ of class $C^{2}$ which satisfies the differential inequality

$$
\Delta v \leqq f(v)
$$

and tends to $\infty$ as $r \rightarrow R$. We shall here use this remark to find explicit upper bounds for certain classes of functions $f$. The following statement also gives a lower bound for $\max u(P)$, which is obtained with the help of a suitable function satisfying the inequality (2).

THEOREM I. Let $f(u)$ be a positive, nondecreasing, differentiable function in $(-\infty, \infty)$, for which

$$
\int_{u}^{\infty} \frac{d t}{f(t)}
$$

exists, and for which

$$
[f(u)]^{1+\lambda} \int_{u}^{\infty} \frac{d t}{f(t)}
$$

is a nondecreasing function of $u$ for some non-negative $\lambda$. If

$$
\phi(r)=\sup _{P \in S_{r}} u(P),
$$

where $u(P)$ ranges over all functions of class $C^{2}$ in $D_{r}$ which satisfy (2), then

$$
\frac{c(\lambda)\left(R^{2}-r^{2}\right)^{2}}{R^{2}} \leqq \int_{\phi(r)}^{\infty} \frac{d t}{f(t)} \leqq \frac{R^{2}-r^{2}}{2 n}
$$

where

$$
c(\lambda)=\frac{1}{4 n} \quad(4 \lambda \leqq n-2)
$$


and

$$
c(\lambda)=\frac{1}{8(2 \lambda+1)}
$$

The left-hand inequality (9) (which yields the upper bound for $\phi(r)$ ) is sharp in the sense that for each number of dimensions $n(n \geqq 2)$, there exists an equation (1) with a spherically symmetric solution $\phi(r)$ for which the sign of equality holds.

The condition that ( 7 ) be a nondecreasing function of $u$ is equivalent to the inequality

$$
f^{\prime}(u) \int_{u}^{\infty} \frac{d t}{f(t)} \leqq 1+\lambda .
$$

It is worth noting that this inequality is always satisfied, for $\lambda=0$, if $\log f(u)$ is a convex function of $u$. Indeed, since $f^{\prime} / f$ is in this case a nondecreasing function of $u$, we have

$$
\frac{1}{f(u)}=\int_{u}^{\infty} \frac{f^{\prime}(t)}{f^{2}(t)} d t \geqq \frac{f^{\prime}(u)}{f(u)} \int_{u}^{\infty} \frac{d t}{f(t)},
$$

and the assertion follows. This implies the following special result.

If $\log f(u)$ is a convex nondecreasing function in $(-\infty, \infty)$, and $\phi(r)$ is defined as before, then

$$
\frac{\left(R^{2}-r^{2}\right)^{2}}{4 n R^{2}} \leqq \int_{\phi(r)}^{\infty} \frac{d t}{f(t)} \leqq \frac{R^{2}-r^{2}}{2 n} .
$$

In particular,

$$
\frac{R^{2}}{4 n} \leqq \int_{\phi(0)}^{\infty} \frac{d t}{f(t)} \leqq \frac{R^{2}}{2 n} .
$$

In the case of a solution $u$ of

$$
\Delta u=e^{u}
$$

which is regular in $D_{R}$, (13) shows that

$$
\log \frac{2 n}{R^{2}-r^{2}} \leqq \phi(r) \leqq 2 \log \frac{2 \sqrt{ } n R}{R^{2}-r^{2}}
$$

and, for $r=0$,

$$
2 \log \frac{\sqrt{ }(2 n)}{R} \leqq \phi(0) \leqq 2 \log \frac{2 \sqrt{ } n}{R}
$$


As already mentioned, the right-hand inequality (15) becomes an equality in the two-dimensional case. For $n \geqq 3$, no explicit solutions of (14) are known. However, it follows from the fact that the substitution of $\rho r$ for $r$ and $u-2 \log \rho$ for $u(\rho>0)$ transforms the equation into itself, that

$$
\phi(0)=2 \log \frac{K_{n}}{R},
$$

where $K_{n}$ is a constant. (16) shows that $\sqrt{ }(2 n) \leqq K_{n} \leqq 2 \sqrt{ } n$. For $n=3$, an improved lower bound for $\phi(0)$ can be obtained from the observation that the 3 -sphere of radius $R$ is contained in the right circular cylinder of the same radius. Hence, $K_{2}<K_{3}$, and thus $2 \sqrt{ } 2<K_{3}$ $<2 \sqrt{ } 3$.

2. Turning now to the proof of the left-hand inequality (9), we consider the function $v=v(r)$ defined by

$$
\frac{c}{R^{2}}\left(R^{2}-r^{2}\right)^{2}=\int_{v}^{\infty} \frac{d t}{f(t)} .
$$

We evidently have $v^{\prime}(0)=0$, and $v(r)$ increases to $\infty$ as $r \rightarrow R$. If we can show that $v$ satisfies the differential inequality (6), it will therefore follow that $\phi(r) \leqq v(r)$, and this will establish the left-hand inequality (9). To verify (6), we write $x$ for any of the variables $x_{1}, \cdots, x_{n}$, and we differentiate (17) twice with respect to $x$. This yields

$$
\begin{aligned}
-\frac{4 c x}{R^{2}}\left(R^{2}-r^{2}\right) & =-\frac{v_{x}}{f(v)} \\
-\frac{4 c}{R^{2}}\left(R^{2}-r^{2}\right)+\frac{8 c x^{2}}{R^{2}} & =-\frac{v_{x x}}{f(v)}+\frac{v_{x}^{2} f^{\prime}(v)}{f^{2}(v)} \\
& =-\frac{v_{x x}}{f(v)}+\frac{16 c^{2} x^{2}}{R^{4}}\left(R^{2}-r^{2}\right)^{2} f^{\prime}(v)
\end{aligned}
$$

Summing over all the $x_{k}$, we obtain

$$
-\frac{4 c n}{R^{2}}\left(R^{2}-r^{2}\right)+\frac{8 c r^{2}}{R^{2}}=-\frac{\Delta v}{f(v)}+\frac{16 c^{2} r^{2}}{R^{4}}\left(R^{2}-r^{2}\right)^{2} f^{\prime}(v),
$$

or, in view of (17),

$$
\frac{\Delta v}{f(v)}=\frac{16 c r^{2}}{R^{2}} f^{\prime}(v) \int_{v}^{\infty} \frac{d t}{f(t)}+\frac{4 n c}{R^{2}}\left(R^{2}-r^{2}\right)-\frac{8 c r^{2}}{R^{2}} .
$$


Condition (12) therefore leads to the inequality

$$
\frac{\Delta v}{f(v)} \leqq 4 c\left[n-\frac{r^{2}}{R^{2}}(n-2-4 \lambda)\right] .
$$

If $4 \lambda \leqq n-2$, it follows that $\Delta v \leqq 4 n c f(v)$, and $v$ will satisfy (6) if $c$ is given the value (10). If $4 \lambda>n-2$, the maximum of the right-hand side of (19) (for $0 \leqq r \leqq R$ ) is attained for $r=R$, and the value (11) for $c$ again leads to a function for which (6) holds.

The sign of equality in (9) will hold if $v$ is a solution of $\Delta v=f(v)$. Since (19) was obtained from (18) by the use of the inequality (12), this is possible only if (12) becomes an equality. This will occur if

$$
f(u)=u^{1+1 / \lambda}, \quad \lambda>0,
$$

and, for $\lambda=0$, if

$$
f(u)=e^{u} .
$$

Furthermore, the right-hand side of (19) will be equal to the constant 1 only if the coefficient of $r^{2}$ vanishes (and, of course, if $c$ is chosen in accordance with (10)). We thus must have $4 \lambda=n-2$. Hence, the left-hand inequality (9) will become an equality in the case of the equation

$$
\Delta u=u^{(n+2) /(n-2),} \quad n \geqq 3,
$$

and, if $n=2$, the equation (14). The solution of (20) obtained in this way is easily confirmed to be of the form

$$
u=\left[\frac{R \sqrt{ }(n(n-2))}{R^{2}-r^{2}}\right]^{8 /(n-2)} .
$$

This, incidentally, seems to be the only $n$-dimensional equation of the form $\Delta u=u^{k}, k>1$, for which a solution can be obtained in terms of elementary functions.

It should be remarked here that, strictly speaking, the equation $\Delta u=u^{k}$ is not covered by Theorem I, since the conditions on $f(u)$ are satisfied only for $u>0$. It is, however, clear that the left-hand inequality (9) will remain valid for solutions of this equation which are positive in $D_{R}$. It is also possible to give a more general version of Theorem I which applies to cases in which the hypotheses on $f(u)$ are satisfied only for $u>\alpha$, where $\alpha$ is a given number. Before we formulate this generalization, we prove the right-hand inequality (9). 
The function $w=w_{p}(r)$ defined by

$$
\frac{\rho^{2}-r^{2}}{2 n}=\int_{w}^{\infty} \frac{d t}{f(t)},
$$

is of class $C^{2}$ in $D_{R}$, and it satisfies the differential inequality (2). Indeed, differentiating with respect to $x=x_{k}$, we obtain

$$
\begin{aligned}
-\frac{x}{n} & =-\frac{w_{x}}{f(w)}, \\
-\frac{1}{n} & =-\frac{w_{x x}}{f(w)}+\frac{w_{x}^{2} f^{\prime}(w)}{f^{2}(w)} \\
& =-\frac{w_{x x}}{f(w)}+\frac{x^{2}}{n^{2}} f^{\prime}(w),
\end{aligned}
$$

and, summing over all the $x_{k}$,

$$
\frac{\Delta w}{f(w)}=1+\frac{r^{2}}{n^{2}} f^{\prime}(w) .
$$

Since $f^{\prime}(w) \geqq 0$, it follows that $\Delta w \geqq f(w)$, and thus, in view of the results quoted above, $w(r) \leqq \phi(r)$. Since $\rho$ may be taken arbitrarily close to $R$, this establishes the right-hand inequality (9). It may also be noted that the only assumption used was $f^{\prime}(u) \geqq 0$; this estimate is therefore valid in the most general case in which the existence of $\phi(r)$ was proved in [3] and [5].

3. We now state the more general version of Theorem I.

ThEOREM II. Let $f(u)$ be continuous in $(-\infty, \infty)$, but satisfy the other hypotheses of Theorem I only for $u>\alpha$, where $\alpha$ is a given number; furthermore, let

$$
\int_{\alpha}^{\infty} \frac{d t}{f(t)}=\infty .
$$

If $u$ is a function of class $C^{2}$ in $D_{r}$ satisfying the inequality (2), and if $P \in S_{r}(r<R)$, then either $u(P) \leqq \alpha$ or, if $u=u(P)>\alpha$,

$$
\frac{c(\lambda)}{R^{2}}\left(R^{2}-r^{2}\right)^{2} \leqq \int_{u}^{\infty} \frac{d t}{f(t)} .
$$

The proof is again based on the fact that the function $v$ defined in (17) tends to $\infty$ for $r \rightarrow R$ and satisfies the inequality (6). Condition 
(21) guarantees that this definition is meaningful for all given values of $R$ and all $r$ in $[0, R)$. The fact that $u \leqq v$, if $u$ satisfies (2), now follows by a slight modification of the argument used in [5]. Since $\Delta v \leqq f(v)$, we have

$$
\Delta(u-v) \geqq f(u)-f(v) .
$$

Suppose there exists a nonempty set $T$ in $D_{R}$ on which $u>v$. Since $v>\alpha$, we necessarily have $u>\alpha$ on $T$, and it follows from our assumptions that $f(u) \geqq f(v)$ on this set. By (23), $u-v$ is therefore subharmonic on $T$. We may assume that $u$ is of class $C^{2}$ on $D_{R}+S_{R}$; this assumption can then be removed by a standard argument. Since $v \rightarrow \infty$ for $r \rightarrow R$, we have $u-v \rightarrow-\infty$ for $r \rightarrow R$, and it follows that the boundary $B$ of $T$ is in $D_{R}$. Hence, $u-v=0$ on $B$, which is absurd, since $u-v$ is positive and subharmonic on $T$. The set $T$ is thus empty, and we must have $u \leqq v$ throughout $D_{R}$. This proves (22).

An immediate consequence of Theorem II is the following result concerning the nonexistence of certain types of entire solutions.

TheOREM III. If $f(u)$ is subject to the hypotheses of Theorem II, and if there exists a function $u$ satisfying (2) which is of class $C^{2}$ in the entire space, then

$$
u(P) ! \leqq \alpha
$$

for all $P$.

Indeed, suppose that $u(P)>\alpha$ for some point $P$. Since (2) remains unchanged under a translation of the coordinate system, we may take $P$ to be the origin. It follows therefore from (22) that

$$
c(\lambda) R^{2} \leqq \int_{u(P)}^{\infty} \frac{d t}{f(t)},
$$

and this produces a contradiction if $R$ is taken large enough.

That entire solutions satisfying (24) may indeed exist is shown by the equation $\Delta u=u^{k+1}$, where $k$ is a positive integer, which satisfies all the assumptions for $\alpha=0$, and which has the trivial entire solution $u \equiv 0$. A nontrivial example is given by the equation

$$
\Delta u=u^{2}+u
$$

in three dimensions, for which $\alpha=0$, and which is known to possess a negative entire solution $[4 ; 6]$.

As a final example, we mention the equation $\Delta u=u^{2 k+1}$, where $k$ is 
a positive integer. Since the equation remains unchanged if $u$ is replaced by $-u$, it follows from Theorem III that, except for the trivial solution $u \equiv 0$, the equation has no entire solutions.

\section{REFERENCES}

1. E. K. Haviland, $A$ note on unrestricted solutions of the differential equation $\Delta u=f(u)$, J. London Math. Soc. 26 (1951), 210-214.

2. E. Hopf, On non-linear partial differential equations, Berkeley Symposium on Partial Differential Equations (Lecture Series), University of Kansas, 1957, pp. 1-31. 510.

3. J. B. Keller, On solutions of $\Delta u=f(u)$, Comm. Pure Appl. Math. 10 (1957), 503-

4. Z. Nehari, On a nonlinear differential equation arising in nuclear physics, Proc. Roy. Irish Acad. Sect. A 62 (1963), no. 9, 117-135.

5. R. Osserman, On the inequality $\Delta u \geqq f(u)$, Pacific J. Math. 7 (1957), 1641-1647.

6. J. L. Synge, On a certain non-linear differential equation, Proc. Roy. Irish Acad. Sect. A 62 (1961), no. 3, 17-41.

7. W. Walter, Über ganze Lösungen der Differentialgleichung $\Delta u=f(u)$, Jber. Deutsch. Math.-Verein. 57 (1955), 94-102.

8. H. Wittich, Ganze Lösungen der Differentialgleichung $\Delta u=e^{u}$, Math. Z. 49 (1943/44), 579-582.

Carnegie Institute of Technology 